
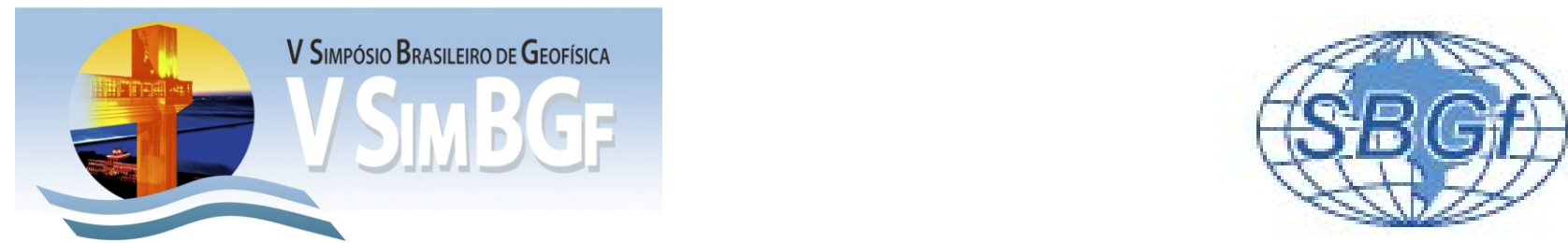

\title{
Representação Tempo versus Frequência do Traço Sísmico utilizando os Métodos de Wigner-Ville e a Decomposição de Fase Mínima
}

Marcus C. B. Andrade - UFBa/IFBa, Milton J. Porsani - CPGG/IGEO/UFBA e INCT-GP/CNPq, Walter E. Medeiros

- UFRN e INCT-GP/CNPq, Bjorn Ursin - Norwegian University of Science and Technology (NTNU)

Copyright 2012, SBGf - Sociedade Brasileira de Geofísica Este texto foi preparado para a apresentação no $V$ Simpósio Brasileiro de Geofísica, Salvador, 27 a 29 de novembro de 2012. Seu conteúdo foi revisado pelo Comitê Técnico do $V$ SimBGf, mas não necessariamente representa a opinião da SBGf ou de seus associados. É proibida a reprodução total ou parcial deste material para propósitos comerciais sem prévia autorização da SBGf.

\section{RESUMO}

A decomposição espectral de dados sísmicos encontra aplicação na caracterização do subsolo e na melhora da resolução de imagens em profundidade. Neste artigo, estaremos comparando duas formas distintas de realização desta decomposição: o método da distribuição de Wigner-Ville(WVD), tomado como referência, e outro método baseado em ondaletas de fase mínima geradas através do filtro de Wiener-Levinson, atuando sobre uma janela móvel - uma decomposição de fase mínima(DFM). O método DFM exibiu características similares ao WVD, contudo possui uma resposta de amplitude no espaço tempo-frequência mais rápida e menor interferência entre frequências próximas, sendo, portanto, uma decomposição promissora para o estudo da propagação de pulsos sísmicos em meios dissipativos.

\section{INTRODUÇÃO}

A decomposição espectral encontra variadas aplicações na análise de dados sísmicos. São exemplos destas aplicações a análise espectral, a filtragem, e, mais recentemente, a caracterização do subsolo e identificação de reservatórios de hidrocarbonetos (Bellefleur et al., 2007; Wu e Liu, 2009). A decomposição espectral pode ser realizada de formas variadas e permite a representação da energia associada a um pulso sísmico no espaço de tempo-frequência (de Castro Nunes et al., 2011; Miao et al., 2007). Neste artigo, estaremos comparando duas formas distintas de realização desta decomposição, o método da distribuição de Wigner-Ville(DWV), tomado como referência, e outro método, baseado em ondaletas de fase mínima extraídas a partir de uma janela móvel usando o filtro de Wiener-Levinson, como proposto em (Porsani et al., 2012). Ambos permitem mapear a energia associada a um pulso sísmico em função do tempo e das frequências de propagação.

Para muitas análises, o modelo de refletividade do solo, o qual despreza outras informações portadas pelo sinal refletido, tais como informações sobre a evolução da forma da onda e do decaimento do pulso devido à variadas fontes de dissipação, é suficiente. Contudo, em estudos de atenuação do pulso sísmico para identificação dos materiais constituintes do subsolo ou para o melhoramento de imagens em profundidade, pelo uso de alguma técnica de restituição da energia dissipada pelo meio de propagação, outros modelos podem se fazer interessantes (Reine et al., 2012).

Na literatura, a atenuação da amplitude de um pulso sísmico é modelada por oscilações com decaimento exponencial, no qual o fator de atenuação, associado ao fator de qualidade, é dependente da frequência (Toverud e Ursin, 2005). Este tipo de atenuação leva a deformações da forma da onda, que, em dados sísmicos reais, dificulta a interpretação geofísica. Adicionalmente, há uma perda constante de energia durante a propagação da onda, energia esta, que, se reposta adequadamente, levaria a uma melhora de resolução nestas profundidades. Ambas as situações apresentadas dependem de técnicas que permitam a análise do sinal no espaço de tempo-frequência, justificando, assim, este estudo comparativo.

A decomposição espectral pelo método WVD, proposta para tratar o problema da decomposição de sinais não estacionários (Martin e Flandrin, 1985), tem como desvantagens energia negativa e aparecimento de interferências devido a termos cruzados. Métodos baseados em janelas móveis têm sido propostos com a intenção de evitar estas dificuldades (Reine et al., 2009). No que segue, serão apresentados os métodos de WVD e DFM e, na sequência, estes métodos serão aplicados a traços sísmicos gerados a partir de dois modelos dissipativos, um com uma camada e outro de duas camadas. Por fim, os resultados serão apresentados, e discutidas as vantagens e desvantagens do método DFM em relação ao WVD.

\section{REPRESENTAÇÃO DO TRAÇO SÍSMICO PELA DISTRIBUIÇÃO DE WIGNER-VILLE}

A distribuição de Wigner-Ville(DWV) de um traço sís- 
mico real $x(t)$ é definida como

$$
W V D(t, f)=\int_{-\infty}^{+\infty} X(t+\tau) \bar{X}(t-\tau) e^{-j 2 \pi f \tau} d \tau
$$

onde $\tau$ é a variável de lapso de tempo, e o traço sísmico real $x(t)$ é associado ao sinal complexo $X(t)$ através de

$$
X(t)=x(t)+j H[x(t)],
$$

onde a componente complexa é definida pela transformada de Hilbert do próprio traço. Observa-se que o produto do sinal complexo e o seu conjugado, com o lapsos de tempo avançado e retardado, respectivamente, permitem a interpretação deste termo como a matrix de autocorrelação da função $X(t)$, ou seja,

$$
R_{X}(t, \tau)=X(t+\tau) \bar{X}(t-\tau) .
$$

A distribuição de Wigner-Ville, então, se apresenta como

$$
W V D(t, f)=\int_{-\infty}^{+\infty} R_{X}(t, \tau) e^{-j 2 \pi f \tau} d \tau,
$$

sendo o lado direito imediatamente identificado como a transformada de Fourrier da autocorrelação do sinal. O efeito da transformada de Wiger-Ville sobre um traço sísmico é distribuir sua energia sobre o plano de tempo e frequência. Como todos os valores do traço participam na determinação de cada valor da DWV ao invés de apenas uma janela do sinal, a DWV localiza a energia no plano tempo e frequência com resolução ótima. Contudo a sua característica bilinear dá origem às interferências associadas aos termos cruzados(ITC) e a valores negativos de energia, dificultando a sua interpretação como o mapa da distribuição de energia no espaço de tempo-frequência (Wu e Liu, 2009).

Os ITCs podem ser demonstrados se considerarmos um sinal composto $x(t)=x_{1}(t)+x_{2}(t)=A e^{j \omega_{1} t}+B e^{j \omega_{2} t}$, que possui WVD dado por

$$
\begin{gathered}
\frac{W V D_{x}(t, \omega)}{2 \pi}=A^{2} \delta\left(\omega-\omega_{1}\right)+B^{2} \delta\left(\omega-\omega_{2}\right) \\
+2 A B \cos \left(\frac{\omega_{1}-\omega_{2}}{2}\right) \delta\left(\omega-\frac{\omega_{1}+\omega_{2}}{2}\right) .
\end{gathered}
$$

O ITC corresponde ao terceiro termo, que aparece no ponto médio entre $\omega_{1}$ e $\omega_{2}$, e possui uma frequência de oscilação dada por $\left(\omega_{1}-\omega_{2}\right) / 2$.

No que segue, estaremos usando a DWV do domínio discreto, o qual recebe a denominação pseudo-distribuição de Wigner-Ville(PDWV), sendo necessário apenas a substituição das integrais por somatórios correspondentes.

\section{REPRESENTAÇÃO DO TRAÇO SÍSMICO PELA DECOMPOSIÇÃO DE FASE MÍNIMA}

Este método se propõe a representar um traço sísmico no espaço tempo-frequência a partir de ondaletas geradas por um filtro de Wiener-Levinson(WL) aplicado sobre uma janela móvel. Uma primeira janela $x^{\prime}(t)$ de tamanho $n$ ( com $n<N$ ) é selecionada a partir do traço sísmico real $x(t)$ de tamanho $N$. O início e o fim da janela são suavizados através do uso de um filtro de Hamming . Para esta janela, calcula-se a autocorrelação

$$
R_{k}=\sum_{k=1}^{n} x_{t} x_{t+k}
$$

onde $k$ corresponde a um lapso de tempo variável, permitindo a obtenção da matriz simétrica de autocorrelação, que satisfaz

$$
\left[\begin{array}{cccc}
R_{o} & R_{1} & \ldots & R_{n} \\
R_{1} & R_{o} & & \vdots \\
\vdots & & \ddots & R_{1} \\
R_{n} & \ldots & R_{1} & R_{o}
\end{array}\right]\left[\begin{array}{c}
g_{1} \\
g_{2} \\
\vdots \\
g_{n}
\end{array}\right]=\left[\begin{array}{c}
E_{g, n} \\
0 \\
\vdots \\
0
\end{array}\right](8)
$$

onde $g$ é denominado de filtro de predição unitária ou de Wiener-Levinson. Impondo-se a condição de normalização sobre o filtro e usando o fato da matriz de autocorrelação ser uma matriz simétrica e bandeada, é possível obter os coeficientes do filtro WL. Para cada janela $\ell$, estes coeficientes podem ser interpretados como uma ondaleta de fase mínima $w^{\ell}$ que porta as principais frequências da porção do traço sobre estudo, sendo que $g^{\ell}$ e $w^{\ell}$ satisfazem

$$
g_{n}^{\ell} * w_{m}^{\ell}=\delta_{m n},
$$

onde $\delta_{m n}$ é o usual delta de Kronecker. Esta ondaleta pode ser usada para recompor o traço sísmico se soubermos o peso com que ela aparece na seção do traço sísmico sobre estudo.

Para este fim, a janela é deslocada ao longo do traço, gerando $N$ ondaletas que são arrumadas ao longo da diagonal principal de uma matrix quadrada $N$, a matriz das ondaletas. Convolvendo esta matriz com um vetor de pesos ou refletividade, é possível restituir-se o traço sísmico. Tem-se, portanto, um sistema

$$
x=W r,
$$

que pode ser invertido para a obtenção do vetor de refletividade $r$, podendo a inversão ser realizada pelo método dos mínimos quadrados. Como a refletividade representa a escala com que a ondaleta participa no traço, passamos a matriz de ondaletas, que porta a evolução das frequências no traço, para o espaço de tempofrequência, através de uma transformada de Fourrier após a devida ponderação de cada ondaleta pela sua refletividade,

$$
W_{p}=\left[r_{1} W_{c 1}, r_{2} W_{c 2}, \ldots, r_{N} W_{c N}\right],
$$

onde $W_{c i}$ é a a coluna $i$ de $W_{p}$. A matrix de ondaletas ponderadas pode ser representada graficamente como 
na Figura 1. Já a visualização gráfica da matriz que resulta da transformada de Fourrier, vista em Figura 3, corresponde à visualização da matriz DWV do método anterior. São estas matrizes que este artigo se propõe a comparar. Neste caso, como a matriz DWV possui van-

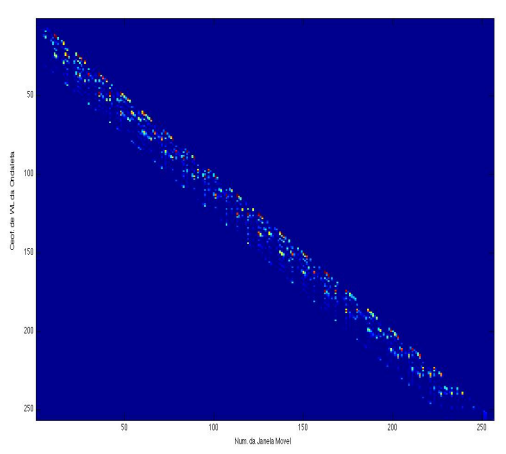

Figura 1: Representação gráfica da matriz diagonal de ondaletas - uma ondaleta por coluna.

tagens e desvantagens conhecidas, esta é tomada como a matriz de referência. O algoritmo DFM é apresentado na Figura 2.

\begin{tabular}{|c|c|c|}
\hline Prepara Janela & 3 & \\
\hline $\begin{array}{l}\text { Calcula Matrix de } \\
\text { Autocorrelação }\end{array}$ & & Fim \\
\hline $\begin{array}{c}\text { Obtém Coeficiente } \\
\text { de WL }\end{array}$ & & Constrói Gráfico \\
\hline \begin{tabular}{|c|} 
Arruma Ondaleta em \\
Matrix Diagonal \\
\end{tabular} & $\square$ & \begin{tabular}{|l|} 
Realiza FFT da Matriz \\
Diagonal Ponderada \\
\end{tabular} \\
\hline$\downarrow$ & & $\uparrow$ \\
\hline \begin{tabular}{|c|} 
Inverte Matriz \\
Diagonal para obter \\
vetor de refletividade
\end{tabular} & $\longrightarrow$ & $\begin{array}{c}\text { Obtém Matriz } \\
\text { Diagonal Ponderada }\end{array}$ \\
\hline
\end{tabular}

Figura 2: Algoritmo do Método DFM.

Observa-se que para a construção dos gráficos referentes ao método DFM, as amplitudes foram elevadas ao quadrado, eliminando os valores negativos, e um filtro de suavização gaussiano foi utilizado.

\section{RESULTADOS}

Os métodos foram testados com traços sintéticos gerados a partir do modelo de Kolsky-Futterman, modelo este que permite um fator de atenuação com dependência linear sobre a frequência do sinal (Varela, 1979). Para uma profunda discussão deste e de outros modelos, ver (Toverud e Ursin, 2005) . Para teste dos algoritmos, foram usados dois cenários distintos. O primeiro consiste em um modelo de uma camada dissipativa com fator de qualidade, frequência e velocidade de propa- gação de referência dados por $\omega_{r}=2000, Q_{r}=50$ e $c_{r}=1000$. O pulso sísmico, obtido por modelagem direta, possui uma única frequência $\omega=700$. Para o segundo cenário, foi utilizado um modelo de duas camadas dissipativas de espessura igual, com $\omega_{r 1}=800$, $Q_{r 1}=500$ e $c_{r 1}=2000$ e com $\omega_{r 2}=1000, Q_{r 2}=400$ e $c_{r 2}=1500$. Neste caso, o pulso sísmico foi composto por duas frequências superpostas com iguais amplitudes, sendo estas $\omega_{1}=1100$ e $\omega_{2}=3000$.

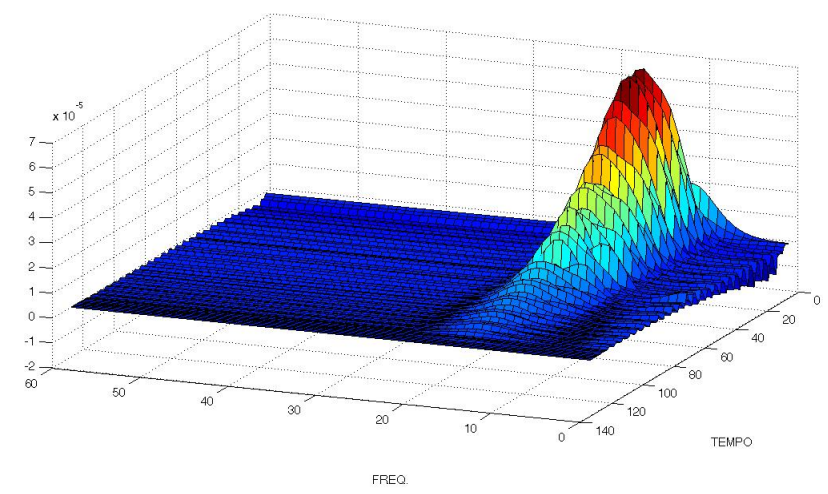

Figura 3: DFM de traço sintético com apenas uma frequência. Observa-se um maior espalhamento da amplitude(eixo vertical) em relação às frequências.

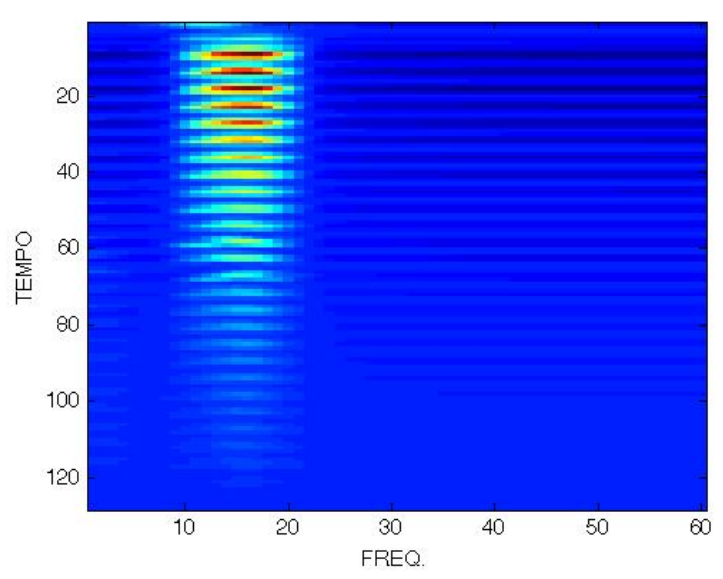

Figura 4: DFM de traço sintético em 2D. O decaimento exponencial do sinal é evidente.

Os gráficos referentes ao método DFM exibidos nas Figuras 3 e 7 foram obtidos usando 11 coeficientes do filtro WL. Adicionalmente, o tamanho da janela móvel para o traço sintético de tamanho igual a 256 foi de 16 valores.

Os gráficos [3] e [5] mostram a decomposição de um traço sísmico, com monofrequência, propagados através de uma única camada dissipativa, usando os métodos DFM e PDWV, respectivamente. Observa-se que a localização da energia é melhor resolvida pelo método PDWV, contudo, neste método, verifica-se um alias de 


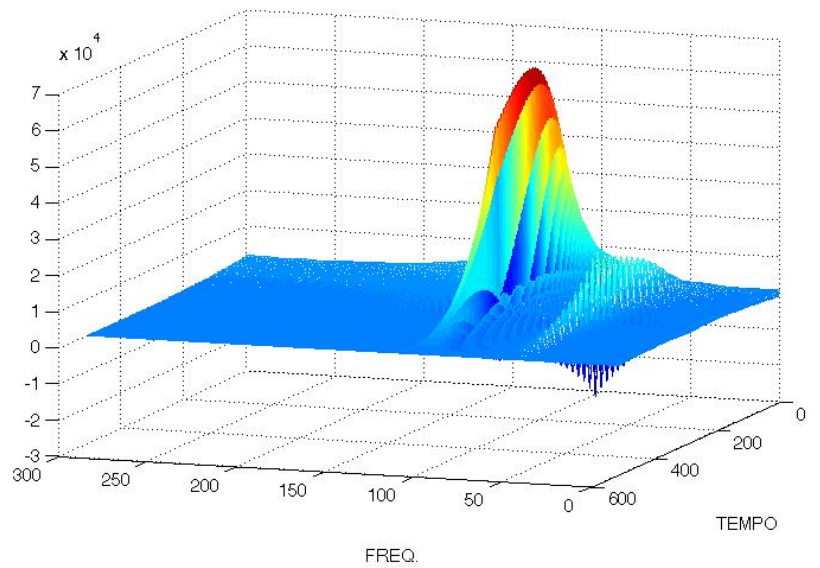

Figura 5: PWVD de traço sintético com apenas uma frequência e presença de alias de menor frequência.

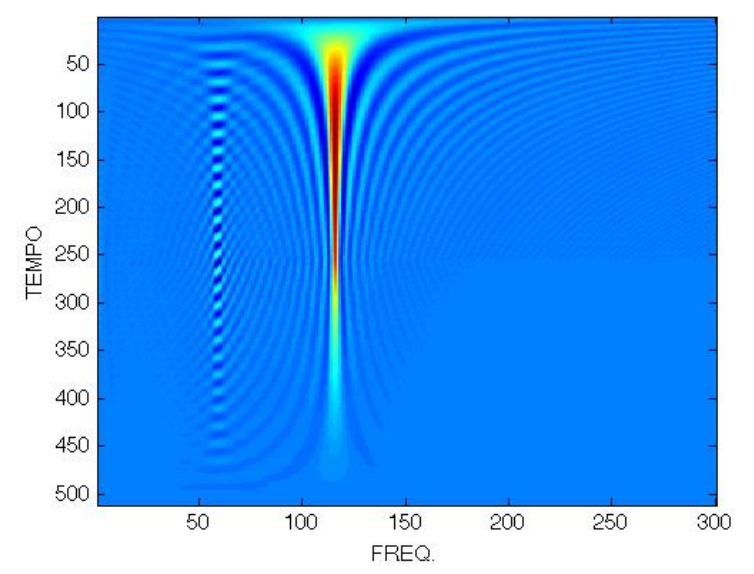

Figura 6: PWVD de traço sintético em 2D. Observa-se a amplitude máxima é alcançada somente para $t \approx 150$.

frequência inferior e um retardo de resposta, a amplitude máxima não é atinginda no início do traço.

São apresentados nas Figuras 7 e 8 a decomposição de traços sísmicos formados por duas frequências superpostas que se propagam através de duas camadas com atenuação distinta, novamente, para os métodos DFM e PDWV, respectivamente. Os ITCs são evidentes na $\mathrm{Fi}$ gura 8 e estão localizados a meia frequência, possuindo amplitudes muito maior que as amplitudes da decomposição do próprio sinal. A Figura 7, para DFM, mostra ITC inexistente ou desprezível. Adicionalmente, é possível verificar o efeito da dissipação mais elevada para frequências mais altas, pois, em ambos os métodos DFM e PWVD, o traço de maior frequência decai mais rapidamente. Isto somente é possível pela resposta rápida do método à variação de energia, localizando a amplitude máxima próxima ao início do sinal. O método DFM evidenciou o decaimento exponencial do sinal transiente de forma superior ao método PWVD. Observa-se que no método DFM foi possível a eliminação dos das amplitudes negativas, facilitando a interpretação dos gráficos como representantes da localização da energia, o mesmo não sendo possível no PWVD devido aos ITCs.

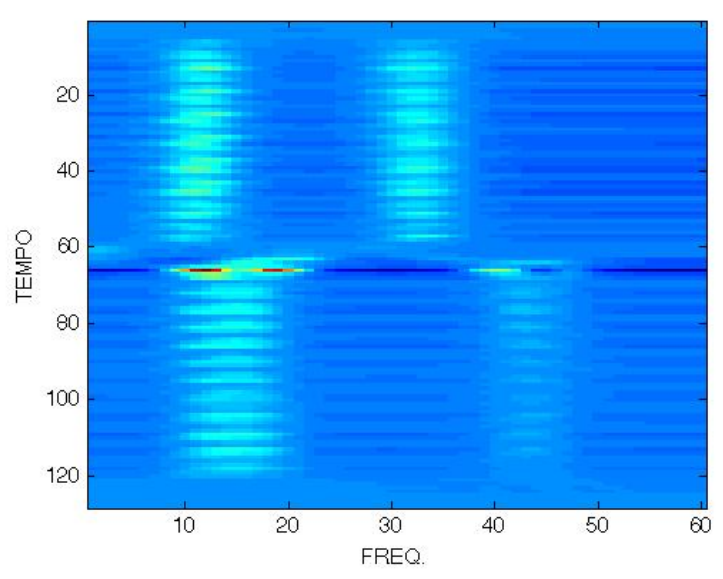

Figura 7: DFM de traço sintético com duas frequências em modelo de duas camadas com $Q$ distintos.

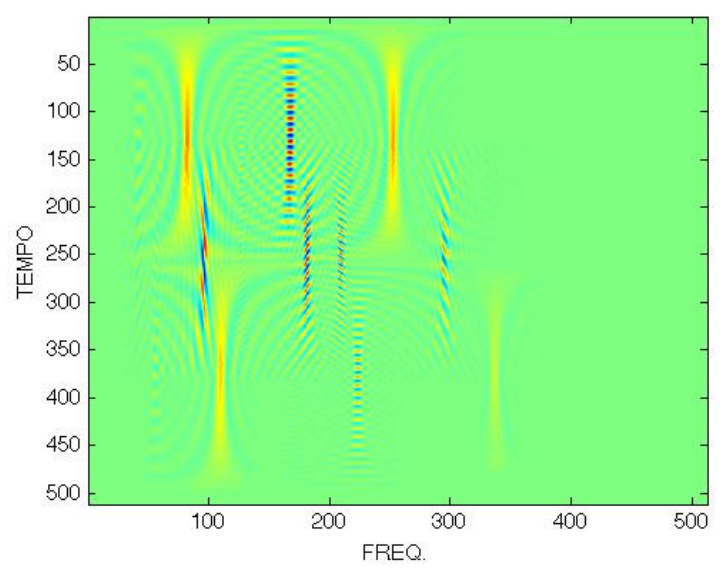

Figura 8: PWVD de traço sintético com duas frequências e presença de ITC acentuado.

\section{DISCUSSÃO E CONCLUSÕES}

As vantagens e desvantagens dos dois métodos espectrais sob estudo, DFM e PWVD, foram expostas. O método de referência, o PWVD, possui conhecidas limitações na representação de sinais multifrequência, o aparecimento dos ITC. Adicionalmente, a variação lenta da amplitude no espaço de tempo-frequência leva a dificuldades na determinação do decaimento do sinal devido à propagação em meios dissipativos. Neste método, a localização e resolução de energia se mostraram ótimas. No método DFM, a decomposição do traço multifrequência não exibiu ITCs acentuados. A resolução no espaço tempo-frequência é satisfatória, mesmo que 
inferior ao método de referência, e muito depende da quantidade de coeficientes de WL que são considerados. A amplitude da energia foi mais bem representada pelo método DFM, ficando a atenuação dissipativa evidenciada nos gráficos associados a este método. A eliminação dos amplitudes negativas e a suavização gaussiana contribuiram para a eliminação da excessiva oscilação nos gráficos do método DFM e facilitaram a localização da energia no espaço tempo-frequência.

\section{AGRADECIMENTOS}

Os autores agradecem o apoio do CPGG/IGEO/UFBA. M. Porsani e W. Medeiros agradecem ao CNPQ pelo apoio ao projeto.

\section{REFERÊNCIAS}

Bellefleur, G., Riedel, M., Brent, T., Wright, F., e Dallimore, S., 2007, Seismic Wave Attenuation in Gas Hydrate-Bearing Sediments from Vertical Seismic Profiling Data: Let it Flow - 2007 CSPG CSEG Convention, pages 598-601.

de Castro Nunes, B. I., Eugênio de Medeiros, W., Farias do Nascimento, A., e de Morais Moreira, J. A., 2011, Estimating quality factor from surface seismic data: $A$ comparison of current approaches: Journal of Applied Geophysics, 75, no. 2, 161-170.

Martin, W., e Flandrin, P., 1985, Wigner-Ville spectral analysis of nonstationary processes: IEEE Transactions on Acoustics, Speech, and Signal Processing, 33, no. $6,1461-1470$.

Miao, X., Todorovic-Marinic, D., e Klatt, T., 2007, Enhancing seismic insight by spectral decomposition: Enhancing seismic insight by spectral decomposition:, Society of Exploration Geophysicists, San Antonio 2007 Annual Meeting, 1437-1441.

Porsani, M. J., Ursin, B., e Silva, M. G., 2012, Recursive estimation of reflectivity by minimum-delay seismic trace decomposition: submitted to Geophysics.

\footnotetext{
Reine, C., van der Baan, M., e Clark, R., 2009, The robustness of seismic attenuation measurements using fixedand variable-window time-frequency transforms: Geophysics, 74, no. 2, WA123.

Reine, C., Clark, R., e Baan, M. V. D., 2012, Robust prestack Q -determination using surface seismic data : Part 1 -Method and synthetic examples:, 77, no. 1 .
}

Toverud, T., e Ursin, B., 2005, Comparison of seismic attenuation models using zero-o set VSP data.: Geophysics, 70, no. 2, F17-F25.

Varela, C. L. U., 1979, Um Estudo da Atenuação e Dispersão: Modelagem Direto e Inverso: Ph.D. thesis, UFBa.

Wu, X., e Liu, T., 2009, Spectral decomposition of seismic data with reassigned smoothed pseudo Wigner-Ville distribution: Journal of Applied Geophysics, 68, no. 3, 386-393. 